\title{
Intuitive Control of Small Flying Robots
}

\author{
Christian Blum, Oswald Berthold, Philipp Rhan, Verena V. Hafner \\ Cognitive Robotics, Department of Computer Science \\ Humboldt-Universität zu Berlin \\ Berlin, Germany
}

\begin{abstract}
In this paper we show a new perspective on human-robot interaction by presenting a system for intuitive interaction with flying robots. This goes beyond the usual remote control of these robots, by having an interactive space where people can physically interact with flying robots in an intuitive and safe way. The presented system has various ways of interaction and provides the prerequisites for many interesting future applications.
\end{abstract}

\section{Keywords}

human-robot interaction, flying robots, intuitive interaction

\section{INTRODUCTION}

When untrained individuals need to interact with one or several autonomous flying systems, an important prerequisite is that the interaction is both intuitive and safe. Examples for such situations arise from applications such as the factory of the future, entertainment or art, and when it is desirable to teach a robot by demonstration. To accomplish this task, the hardware should be easy to handle, safe and allow for dynamic and intuitive human-robot interaction.

One approach is that followed in $[5,7]$ providing a safety layer between the untrained operator and the robot. Intrinsic safety in particular is a major issue with flying robots due to potential crashes and contact with propellers. Regarding direct interaction, a vision-mediated gestural interface was proposed in [6] and a blimp for safe interaction with and safe operation in the vicinity of crowds is described in [3], but the vehicle is rather bulky. An approach for reducing danger of self-destruction for the robot itself is described in $[4,1]$. One general way of reducing the potential for harm is to reduce the size and mass of such robots. Here we present a system using very lightweight $(\mathcal{O}(50 \mathrm{~g}))$ quadrotor helicopters which can be used for indoor interaction experiments.

While it is currently quite challenging to realize full onboard sensing and behaviour generation (control) of very small quadrotors [2], results from the proposed experiments

Permission to make digital or hard copies of part or all of this work for personal or classroom use is granted without fee provided that copies are not made or distributed for profit or commercial advantage, and that copies bear this notice and the full citation on the first page. Copyrights for third-party components of this work must be honored. For all other uses, contact the owner/author(s). Copyright is held by the author/owner(s).

HRI'14, March 3-6, 2014, Bielefeld, Germany.

ACM 978-1-4503-2658-2/14/03.

http://dx.doi.org/10.1145/2559636.2559826.

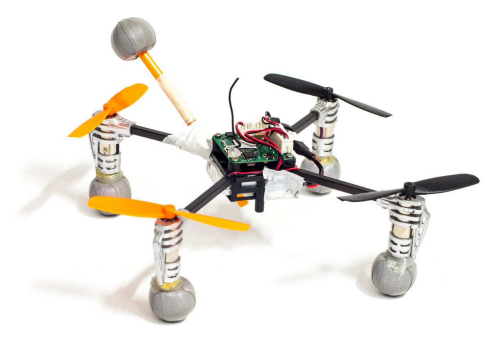

Figure 1: Modified Walkera Ladybird with markers for the tracking system.

can easily be transferred from the tracking system case to the full onboard case because these results relate to general features of robot behaviour.

\section{SYSTEM}

As discussed above we chose a small quadrotor, the popular toy Walkera Ladybird ${ }^{1}$. The manufacturer designates it to be safe for children from the age of 14 . It weighs only $31 \mathrm{~g}$ (with markers) and has a diameter of approx. $15 \mathrm{~cm}$ making it inherently safe even when accidentally hitting the human. We added five markers as can be seen in Fig. 1 to enable $6 \mathrm{D}$ tracking. In addition to human safety, the weight and size of the robot also make it almost immune to crashes etc.

Controlling a small flying robot in the direct vicinity of a human and facilitating interaction requires a closed-loop system in order to enable intuitive and safe behaviour of the robot. Weight constraints on a small robot are severe, thus we opted for external control and sensors.

We track the copter using an external tracking system consisting of six Natural Point OptiTrack Flex13 Cameras yielding $6 \mathrm{D}$ positions of the robot at $120 \mathrm{~Hz}$ with an accuracy of about $0.5 \mathrm{~mm}$ and a latency of less than $10 \mathrm{~ms}$. These positions are streamed over ethernet to the computer controlling the robot using a Natural Point protocol.

The robot is controlled using the MagicCube remote by Walkera which is controlled by an Arduino ${ }^{2}$, which is attached to the control computer. The computer receives $6 \mathrm{D}$ positions from the tracking system and sends motor com-

\footnotetext{
${ }^{1}$ http://www.walkera.com/

${ }^{2}$ https://github.com/koro/mCube
} 


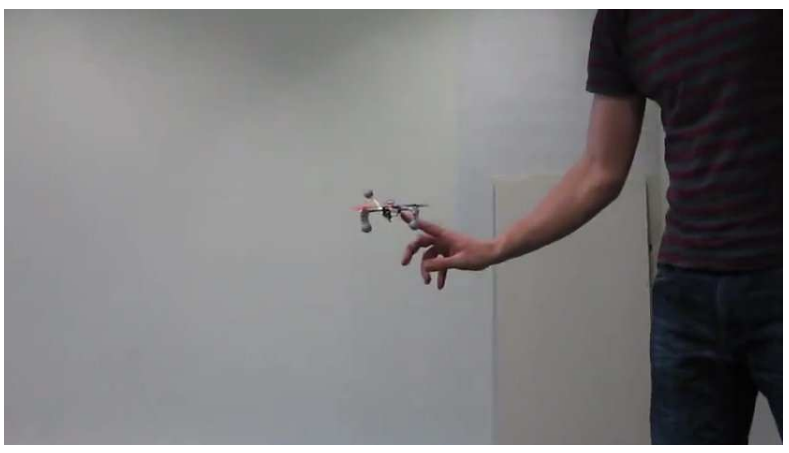

Figure 2: Human robot interaction by direct physical interaction, in this case mediated by pushing.

mands to the robot. We use our Mavhub ${ }^{3}$ infrastructure to deal in quasi-real-time with the control. At the moment the robot is controlled by simple PID controllers.

To enhance the safety of bystanders and other scientists not participating in the experiments, we implemented a virtual safety cage. The main function of this cage is to shut down the motors of the robot when it leaves the cage by accident. In contrast to other indoor experiments with flying robots we do not need a physical safety cage or net because of the small size of the robot.

\section{INTERACTION}

Our vision is to facilitate interaction between flying robots and humans, that goes beyond device-based remote control but instead emphasizes direct interaction with the robot. This provides a basis for working together with the robot, teaching it by demonstration and physically correcting its mistakes.

\subsection{Direct Physical Interaction}

This is not only the most intuitive way of interacting with a robot, it also does not require additional hardware, as opposed to also tracking the human or using an RGB-D camera. For this to work, safety is imperative in order to ensure the health of the human and the physical integrity of the robot. An example interaction is depicted in Fig. 2.

We can use the error as determined by our control loop to detect sufficiently large offsets in position, which are due to external forces inflicted on the system by physical contact. A more sensitive alternative is the use of more advanced internal models, and detecting the interaction by monitoring the prediction errors of the forward model. These methods allow us to change the setpoints of the controllers and to freely position the robot in $3 \mathrm{D}$ space.

\subsection{Kinesthetic Teaching}

The next logical thing to do after changing the setpoints of the robot's position controller, is teaching it trajectories, of which an example is depicted in Fig. 3. For this, there are two intuitive possibilities.

Using the method of direct physical interaction between the human and the robot as described above, it is possible to not only change the setpoint but to demonstrate a whole trajectory. For this the robot has to recognize the beginning

\footnotetext{
${ }^{3}$ https://github.com/calihem/mavhub
}

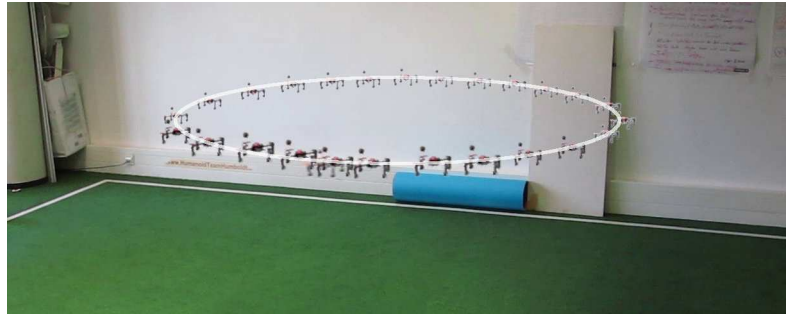

Figure 3: The robot follows an exemplary trajectory.

and the end of the interaction, which can be indicated by motion signatures. Knowledge about its position between these two points in time defines the trajectory. Another method is to use an additional marker within the tracking system and use it to record a trajectory demonstration. This can be advantageous in cases where the trajectory is more complex and using the copter directly would be too tiring or complex for the human.

\section{SUMMARY AND FUTURE WORK}

The proposed system provides a building block for solving more complex cooperation tasks for untrained individuals working with flying robots. These can be imitation learning, refinement of motion primitives or orchestrating robot teams for complex industrial tasks. Future work will focus on augmenting interaction detection and iterative interactive refinement of demonstrated trajectories. This can include use of additional sensors to enhance safety of the overall system and situational awareness of the robot.

\section{ACKNOWLEDGMENTS}

This work was funded in part by the DFG Research Training Group METRIK.

\section{REFERENCES}

[1] A. Briod et al. Contact-based navigation for an autonomous flying robot. In International Conference on Intelligent Robots and Systems (IROS'13), 2013.

[2] A. Briod, J.-C. Zufferey, and D. Floreano. Optic-flow based control of a $46 \mathrm{~g}$ quadrotor. In Workshop on Vision-based Closed-Loop Control and Navigation of Micro Helicopters in GPS-denied Environments, IROS'13, 2013.

[3] M. Burri et al. Design and control of a spherical omnidirectional blimp. In Proc. IROS'13, 2013.

[4] A. Klaptocz et al. Euler Spring Collision Protection for Flying Robots. In Proc. IROS'13, 2013.

[5] D. Lee et al. Semiautonomous haptic teleoperation control architecture of multiple unmanned aerial vehicles. Mechatronics, IEEE/ASME Transactions on, 18(4):1334-1345, 2013.

[6] V. M. Monajjemi et al. HRI in the Sky: Creating and Commanding Teams of UAVs with a Vision-mediated Gestural Interface. In Proc. IROS'13, 2013.

[7] D. Pitman and M. L. Cummings. Collaborative Exploration with a Micro Aerial Vehicle: A Novel Interaction Method for Controlling a MAV with a Hand-Held Device. Advances in Human-Computer Interaction, 2012. 